\title{
Oesophageal manometry: how well does it predict oesophageal function
}

\author{
C O H RUSSELL AND G WHELAN
}

From the Department of Surgery, Prince Henry's Hospital and Department of Medicine, St. Vincent's Hospital, Melbourne, Australia

SUMMARY The variability in manometric measurements of oesophageal peristalsis was assessed in 10 volunteers. The amplitude, velocity and duration of the peristaltic waves resulting from 10 separate $10 \mathrm{ml}$ boluses of water were measured at fixed distances above the lower oesophageal sphincter (LOS). After a 10 minute rest period with the manometry catheter still in situ peristaltic values in response to a second group of $1010 \mathrm{ml}$ boluses were measured. The measurement of peristaltic amplitude at a fixed distance above the LOS showed wide interindividual variation - for example, at $8 \mathrm{~cm}$ above the LOS the variation between individuals was marked $(\mathrm{p}<0 \cdot 001)$. At the same site, however, there was only a small intra individual variation noted with time $(p>0 \cdot 25)$. Similar differences were noted at 16 and $4 \mathrm{~cm}$ above the LOS. For the first group of swallows, while the interindividual variation remained high $(\mathrm{p}<0.025)$, the measurement variation from site to site was of lesser magnitude $(p>0 \cdot 1)$. Similar findings were noted for interindividual variation and site to site variation at the second group of swallows. The values for velocity showed a similar pattern of variation. From this study we conclude that manometric measurements can be used as a valid method for assessing the effects of drugs on peristalsis in individuals provided all measurements are made at the exact same level in the oesophagus and 'normal' subjects with a large inherent variation are excluded. Any studies comparing peristaltic values in different population groups - for example, normal $v$ reflux patients, may not detect any significant difference even with large sample numbers. Any differences detected may be of no clinical importance because of the large normal variation.

Oesophageal manometry is the gold standard for assessing oesophageal motor function. The data recorded at oesophageal manometry give information on the percentage of observed swallows that result in a 'peristaltic' wave - that is, a wave that is propagated along the entire length of the oesophagus. The amplitude, velocity and duration of that peristaltic wave can be measured at various levels in the oesophagus. The presence or absence of sphincter tone and the degree of inhibition in response to swallowing can also be assessed.

Address for correspondence: C O H Russell, Department of Surgery, Prince Henry's Hospital, St Kilda Road, Melbourne, Victoria 30(04, Australia.

Received for publication 10 December 1986.
Most motility disorders are diagnosed by the presence or absence of peristalsis and the sphincter response to swallowing. Pope in 1970 studied the variation in amplitude of the peristaltic waves recorded from a section of the normal population and found this to be large.'

Analysis of this variation confirmed a large variation between individuals. He concluded 'measurement (of peristaltic amplitude) will frequently not serve to separate normal from abnormal'. Since that study, low compliance infusion systems have replaced the syringe pump and led to greater sensitivity in measuring pressure events. ${ }^{2}$ The purpose of this study was to assess the variations of amplitude, velocity and duration within a normal population using modern low compliance manometry equipment. 


\section{Methods}

SUBJECTS

Ten normal male volunteers (aged 26-64 years; median age 33) with no history of gastrointestinal disease and of similar height $(168-175 \mathrm{~cm})$ were studied following a three hour fast. Peristalsis was recorded via a multilumen catheter assembly incorporating a Dent sleeve. ${ }^{3}$ The outside diameter of the swallowed assembly was $5 \mathrm{~mm}$ and the internal diameter of each perfused lumen was $0.58 \mathrm{~mm}$ at the distal end (swallowed portion) and $1 \mathrm{~mm}$ at the proximal end. Side holes were circumferentially placed $4 \mathrm{~cm}$ apart. Each polyvinyl catheter was perfused with water $(0.6 \mathrm{ml} / \mathrm{min})$ by a hydropneumatic infusion pump. ${ }^{+}$Oesophageal intraluminal pressure changes were detected by four Hewlett Packard (HP) quartz transducers and recorded on heat sensitive paper on a HP recorder. This system provided a response rate $>200 \mathrm{mmHg} / \mathrm{S}$ when the catheter side holes were occluded. Before each study the machine was calibrated against known pressures generated by a hand pump and displayed on an accurate pressure gauge.

The catheter assembly was passed by the nasal route without local anaesthetic and the midpoint of the sleeve was sited in the lower oesophageal sphincter (LOS) before securing the whole assembly on the nose with adhesive tape. After catheter insertion there followed a five minute rest period before the first group of swallows (group A) was recorded. A further rest period of 10 minutes (with the catheter in situ) preceded a second group of swallows (group B). Each swallow recorded was in response to a $10 \mathrm{ml}$ bolus of water, introduced to the mouth by a syringe. There was a $30 \mathrm{~s}$ 'rest' between swallows. The amplitude and duration of each peristaltic wave was measured at three sites in each volunteer $-16,8$, and $4 \mathrm{~cm}$ above the LOS. The velocity of the wave between the points $16 \mathrm{~cm}$ and $8 \mathrm{~cm}$; and $8 \mathrm{~cm}$ and $4 \mathrm{~cm}$ above the LOS was calculated. Amplitude was calculated as the pressure rise from baseline oesophageal pressure (point $\mathrm{A}$ in the Figure) to peak pressure (point $C$ in the Figure). Duration was calculated as the time from the onset of the peristaltic upstroke (point B in the Figure) to the end of the downstroke (point $D$ in the Figure) defined as the point where the wave crossed the level of the preswallow baseline pressure. Velocity was distance divided by the time in seconds between the onset of the upstroke of the peristaltic wave at the two points for example, eight divided by the time elapsed between point $B$ and point $E$ in the Figure.

STATISTICAL ANALYSIS

The data for amplitude, velocity and duration were analysed using analysis of variance (ELF ANOVA module) to determine the contribution which could be made to variation caused by subjects; variation between subjects, as well as the variation because of the site of measurement within the oesophagus. The coefficient of variation was calculated for each subject's measurements in order to compare the consistency of each subject's measurements from site to site, individual to individual, and groups of swallows before and after the 10 minute rest period.

\section{Results}

All $10 \mathrm{ml}$ swallows in all volunteers were associated with a fully propagated peristaltic wave - that is, $100 \%$ peristaltic response. Lower oesophageal sphincter relaxation was seen to occur in response to all these swallows.

The mean amplitude values $( \pm S D)$ for each subject; for each of the three sites of measurement $(16,8$, and $4 \mathrm{~cm}$ above the LOS) and for both groups of swallows (A and B) are shown in Table 1. The
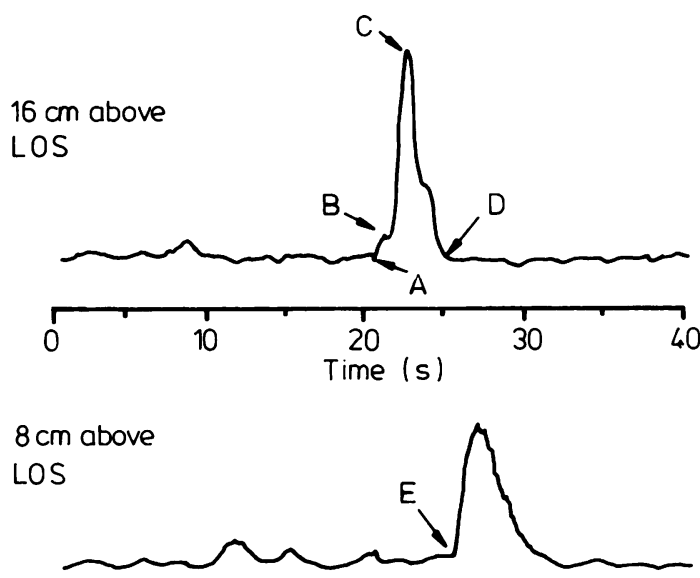

$4 \mathrm{~cm}$ above LOS

Figure Manometry tracing from normal volunteer with amplitude in $\mathrm{mm} \mathrm{Hg}$ on vertical axes and time in seconds on horizontal axes. From top to bottom are seen the pressure events at $16 \mathrm{~cm}, 8 \mathrm{~cm}$, and $4 \mathrm{~cm}$ above the lower oesophageal sphincter (LOS). Points $B$ and $E$ are the onset of a peristaltic wave at 16 and $8 \mathrm{~cm}$ above the $L O S$ respectively. Point $A$ is baseline oesophageal pressure; $C$ is peak amplitude and $D$ represents point of completion of peristaltic wave. 
Table 1 Mean amplitude in $\mathrm{mmHg} \pm S D$ of peristaltic waves from both groups of $10 \mathrm{ml}$ wet swallows in 10 normal volunteers (group B values shown in parentheses)

\begin{tabular}{lccc}
\hline Subject & $\begin{array}{l}\text { l6cmabove } \\
\text { LOS }\end{array}$ & $\begin{array}{l}8 \mathrm{cmabove} \\
\text { LOS }\end{array}$ & $\begin{array}{l}\text { 4 cmabove } \\
\text { LOS }\end{array}$ \\
\hline 1 & $40 \cdot 9 \pm 4 \cdot 9$ & $69 \cdot 3 \pm 10 \cdot 2$ & $35 \cdot 6 \pm 10 \cdot 8$ \\
& $(48 \cdot 2 \pm 7 \cdot 6)$ & $(77 \cdot 3 \pm 10 \cdot 6)$ & $(43 \cdot 5 \pm 8 \cdot 2)$ \\
2 & $37 \cdot 8 \pm 10 \cdot 4$ & $53 \cdot 2 \pm 19 \cdot 7$ & $32 \cdot 4 \pm 9$ \\
& $(39 \cdot() \pm 7 \cdot 8)$ & $(46 \cdot 2 \pm 16 \cdot())$ & $(26 \cdot 1 \pm 5 \cdot 3)$ \\
3 & $113 \cdot 0 \pm 8 \cdot 3$ & $72 \cdot 6 \pm 30 \cdot 4$ & $97 \cdot 7 \pm 32 \cdot 8$ \\
& $(118 \cdot 4 \pm 8 \cdot 8)$ & $(87 \cdot 1 \pm 25 \cdot 8)$ & $(99 \cdot 9 \pm 17 \cdot 5)$ \\
4 & $97 \cdot 3 \pm 43 \cdot 4$ & $156 \cdot 0 \pm 53 \cdot 0$ & $149 \cdot 0 \pm 60 \cdot 4$ \\
& $(145 \cdot 5 \pm 39 \cdot 3)$ & $(177 \cdot 5 \pm 35 \cdot 7)$ & $(121 \cdot 0 \pm 40 \cdot 7)$ \\
5 & $24 \cdot 2 \pm 9 \cdot 1$ & $96 \cdot 2 \pm 34 \cdot 5$ & $92 \cdot 2 \pm 34 \cdot 1$ \\
& $(31 \cdot 0 \pm 12 \cdot 8)$ & $(92 \cdot 0 \pm 31 \cdot 4)$ & $(119 \cdot 5 \pm 24 \cdot 3)$ \\
6 & $38 \cdot 6 \pm 5 \cdot 6$ & $112 \cdot 8 \pm 4 \cdot 1$ & $80 \cdot 6 \pm 12 \cdot 1$ \\
& $(40 \cdot 8 \pm 10 \cdot 8)$ & $(113 \cdot 9 \pm 7 \cdot 2)$ & $(101 \cdot 8 \pm 17 \cdot 7)$ \\
7 & $87 \cdot 3 \pm 4 \cdot 1$ & $98 \cdot 1 \pm 22 \cdot 0$ & $87 \cdot 3 \pm 33 \cdot 2$ \\
& $(85 \cdot 5 \pm 5 \cdot 2)$ & $(104 \cdot 0 \pm 16 \cdot 7)$ & $(107 \cdot 0 \pm 23 \cdot 6)$ \\
8 & $133 \cdot 3 \pm 11$ & $66 \cdot 8 \pm 26 \cdot 8$ & $100 \cdot 4 \pm 27 \cdot 9$ \\
& $(129 \cdot 0 \pm 9 \cdot 4)$ & $(79 \cdot 6 \pm 8 \cdot 8)$ & $(79 \cdot 6 \pm 25 \cdot 4)$ \\
& $22 \cdot 5 \pm 4 \cdot 9$ & $133 \cdot 9 \pm 24 \cdot 0$ & $29 \cdot 9 \pm 6 \cdot 2$ \\
9 & $(21 \cdot 8 \pm 4 \cdot 7)$ & $(127 \cdot 5 \pm 24 \cdot 1)$ & $(49 \cdot 6 \pm 16 \cdot 0)$ \\
& $104 \cdot 9 \pm 8 \cdot 2$ & $125 \cdot 3 \pm 14 \cdot 8$ & $131 \cdot 9 \pm 11 \cdot 6$ \\
10 & $(97 \cdot 3 \pm 4 \cdot 3)$ & $(131 \cdot 0 \pm 13 \cdot 1)$ & $(128 \cdot 5 \pm 22 \cdot 6)$ \\
& & &
\end{tabular}

overall mean amplitudes $( \pm \mathrm{SD})$ from all 10 subjects - that is, the mean of 200 swallows, was 72.8 $( \pm 10.7) ; 101.0( \pm 11.9)$ and $85.7( \pm 13.7) \mathrm{mmHg}$ at 16,8 , and $4 \mathrm{~cm}$ above the LOS respectively.

The coefficient of variation for amplitude ranged from $3.6 \%$ to $41.9 \%$ (mean $22.1 \%$, median $21.7 \%$ ). For most individuals it was reasonably constant from one group of swallows to another but was more variable from site to site.

Mean velocity values $( \pm S D)$ for each subject for each group of swallows are shown in Table 2. The overall mean velocity $( \pm S D)$ was $2.4 \mathrm{~cm} / \mathrm{s}( \pm 0.2)$ and $1.9 \mathrm{~cm} / \mathrm{s}( \pm 0.5)$ between 16 and $8 \mathrm{~cm}$ and 8 and $4 \mathrm{~cm}$ above the LOS respectively.

The coefficient of variation for velocity ranged from $3 \cdot 7$ to $76 \cdot 7 \%$ (mean $20.0 \%$, median $20.9 \%$ ).

Mean duration values for each subject have not been tabulated but the overall mean duration of the 200 swallows $( \pm S D)$ were $3 \cdot 2( \pm 0 \cdot 6) ; 3 \cdot 8( \pm 0 \cdot 9)$ and $3 \cdot 8( \pm 1 \cdot 0)$ seconds at 16,8 , and $4 \mathrm{cms}$ above the LOS respectively.

The coefficient of variation for duration ranged from 10.3 to $39.5 \%$ (mean $22 \%$, median $21.8 \%$ ).

STATISTICAL ANALYSIS

Amplitude

Examination of Table I reveals that values for amplitude vary considerably between subjects at each site tested (16, 8, and $4 \mathrm{~cm}$ above the LOS). In
Table 2 Mean velocity $\pm S D$ in $\mathrm{cm} / \mathrm{s}$ of peristaltic waves for both groups of $10 \mathrm{ml}$ swallows (group B values in parentheses) in 10 normal volunteers

\begin{tabular}{lcc}
\hline Subject & $\begin{array}{l}\text { Velocity between } \\
16 \text { and } 8 \text { cmabove LOS }\end{array}$ & $\begin{array}{c}\text { Velocity between } \\
8 \text { and } 4 \text { cmabove LOS }\end{array}$ \\
\hline 1 & $2 \cdot(0 \pm 0 \cdot 1$ & $1 \cdot 5 \pm 0 \cdot 4$ \\
& $(1 \cdot 8 \pm 0 \cdot 2)$ & $(1 \cdot 8 \pm 0 \cdot 3)$ \\
2 & $2 \cdot 7 \pm 0 \cdot 1$ & $2 \cdot 1 \pm 1 \cdot 1$ \\
& $(2 \cdot 5 \pm 0 \cdot 2)$ & $(3 \cdot 0 \pm 2 \cdot 3)$ \\
3 & $1 \cdot 7 \pm 0 \cdot 2$ & $2 \cdot 2 \pm 1 \cdot 0$ \\
& $(1 \cdot 7 \pm 0 \cdot 2)$ & $(1 \cdot 7 \pm 0 \cdot 3)$ \\
4 & $2 \cdot 2 \pm 0 \cdot 8$ & $1 \cdot 5 \pm 0 \cdot 7$ \\
& $(3 \cdot 6 \pm 0 \cdot 6)$ & $(0 \cdot 6 \pm 0 \cdot 2)$ \\
5 & $2 \cdot 2 \pm 0 \cdot 5$ & $2 \cdot 6 \pm 0 \cdot 8$ \\
& $(1 \cdot 9 \pm 0 \cdot 2)$ & $(2 \cdot 3 \pm 0 \cdot 7)$ \\
6 & $3 \cdot 1 \pm 0 \cdot 3$ & $1 \cdot 6 \pm 0 \cdot 2$ \\
& $(2 \cdot 7 \pm 0 \cdot 2)$ & $(1 \cdot 4 \pm 0 \cdot 2)$ \\
7 & $3 \cdot 3 \pm 0 \cdot 3$ & $1 \cdot 2 \pm 0 \cdot 3$ \\
& $(3 \cdot 6 \pm 0 \cdot 5)$ & $(1 \cdot 1 \pm 0 \cdot 1)$ \\
8 & $1 \cdot 7 \pm 0 \cdot 3$ & $3 \cdot 0 \pm 0 \cdot 6$ \\
& $(2 \cdot 0 \pm 0 \cdot 1)$ & $(2 \cdot 1 \pm 0 \cdot 3)$ \\
9 & $1 \cdot 6 \pm 0 \cdot 2$ & $1 \cdot 5 \pm 0 \cdot 3$ \\
& $(1 \cdot 6 \pm 0 \cdot 2)$ & $(6 \pm 0 \cdot 2)$ \\
10 & $2 \cdot 7 \pm 0 \cdot 3$ & $2 \cdot 9 \pm 0 \cdot 4$ \\
& $(2 \cdot 6 \pm 0 \cdot 3)$ & $(2 \cdot 4 \pm 0 \cdot 4)$ \\
& &
\end{tabular}

some subjects mean values were approximately three times that of others.

At $16 \mathrm{~cm}$ and $4 \mathrm{~cm}$ the interaction between subject and site was a significant cause of variation. This created difficulty in the precise interpretation of the separate effects of each variable. The major source of variation, however, was between subjects not between sites.

At $8 \mathrm{~cm}$ above the LOS where subject/site interaction was trivial the interindividual component of variation was large $(\mathrm{F} 9,9=48.51 ; \mathrm{p}<0 \cdot 001)$ while the intraindividual variation between groups of swallows was insignificant $(F 1,9=1.69 ; \mathrm{p}>0 \cdot 25)$.

Likewise for either group A or group B swallows site to site variation was insignificant (group $A-F 2$, $18=2 \cdot 23 ; p>0 \cdot 1$ : and group $B-F 2,18=2 \cdot 37 ; p>0 \cdot 1)$ while interindividual variation was prominent (group A - F9, 18=3.01; $\mathrm{p}<0.025$ and group B $-\mathrm{F}$, $18=3 \cdot 67 ; \mathrm{p}<0 \cdot 01)$.

\section{Velocity}

Variabililty caused by interindividual variation (F9, $9=5.54 ; \mathrm{p}<0.01)$ was again greater than that observed for intraindividual variability between groups of swallows $(\mathrm{F} 1,9=0 \cdot 25 ; \mathrm{p}>0 \cdot 25)$.

\section{Duration}

A similar situation was noted - interindividual variation at $8 \mathrm{~cm}$ was prominant $(\mathrm{F} 9,9=5 \cdot 24$; 
$\mathrm{p}<0.025)$ while intraindividual variability was insignificant $(F 1,9=0 \cdot 35 ; p>0 \cdot 25)$.

Frequency distribution histograms were constructed for values of amplitude at each site tested to determine the nature of the distribution. While values for individual subjects were not normally distributed, very few were skewed. The pooled observations at $8 \mathrm{~cm}$, however, where individual/site interaction was insignificant, were near normally distributed.

\section{Discussion}

We have analysed the observed variations in the dimension of oesophageal peristalsis as measured by modern manometric methods (amplitude, velocity and duration of the wave) in a group of 10 asymptomatic 'normal' volunteers. A large interindividual variation in values was found. The effect of the other two variables in the study - site of measurement and time - was difficult to interpret because of the considerable interaction between subject and site as well as between subject and time. At the site where no interaction occurred, however, $(8 \mathrm{~cm}$ above the LOS) a wide variation between individuals was noted (F9, 9=48.01; $\mathrm{p}<0.001)$ while no significant intraindividual variation with time was seen, $(\mathrm{F} 1,9=1.69$; $p>0 \cdot 25$ ). Thus interindividual variation was the major source of variance.

While the population sample is relatively small ( 10 subjects) the number of observations per subject was large. The variability in mean values for amplitude from individual to individual was at least three-fold while for any one individual at any one site mean values varied less than 1-2-fold between groups of swallows.

These observations confirm those of Pope' who noted wide variation between normal subjects. With more modern equipment our mean values are higher ( $95 \%$ confidence interval $93.5-102.65 \mathrm{mmHg}$ ) but without significant intraindividual variation. Unlike Pope we found no significant site to site variation but it should be noted that we sampled fewer sites within the oesophagus.

When other relatively comparable series of manometric values are reviewed ${ }^{-11}$ some differences in the mean values obtained are seen. These differences may be because of the manometric methods used or might even reflect differences in the populations studied. Although both these factors may play a role we suggest these differences are further evidence for there being a wide range of normal values. It is also obvious that each oesophageal laboratory must study its own 'normal population', as adoption of another's range of normal values could be an inappropriate assumption. We question the practice of defining abnormality on the basis of measurements of amplitude, velocity and duration lying outside the mean $\pm 2 \mathrm{SD}$ (or even $\pm 3 \mathrm{SD}$ ) range. We constructed frequency distribution histograms for amplitude, velocity and duration and those indicated that only at the mid-oesophageal site $(8 \mathrm{~cm}$ above the LOS) was there a pattern that was near normal. It is also important to note that this is the only site where the interaction between site and subject was trivial, allowing us to see the marked importance of interindividuality compared with the more minor effect of intraindividual variation. As the actual range quoted will vary considerably depending upon the subjects chosen we may ask whether there is a range of values of amplitude, velocity and duration which can reliably be used to predict whether oesophageal function is normal or abnormal?

It is interesting that normal oesophageal function occurs within such a wide range of values of peristaltic amplitude, velocity and duration. This suggests that in an individual none of these measurements when expressed as an absolute value will reliably predict that individual's function. Would function be better predicted by the product of these values - for example, amplitude $\times$ duration or amplitude $\times$ velocity. Some initial efforts showed that such manipulations only served to increase the range of values. There is no doubt, however, that qualitative peristaltic abnormalities are found when dysphagic patients are studied with manometry. These range from complete absence of a peristaltic wave to simultaneous contractions (analagous to ventricular fibrillation). There are findings between these extremes where values outside a quoted range of normal values are taken as being indicative of abnormality - for example, nutcracker oesophagus." When these patients are studied by a further independent method of analysing function - radionuclide transit - by no means all are shown to have a functional abnormality. ${ }^{12} 13$ This may be supportive evidence that values outside the normal range may not always be associated with a functional abnormality. There must obviously be the situation where a patient with dysphagia may have identical values for amplitude, velocity and duration, to an asymptomatic, and supposedly normal subject.

Looking at the corollary to this situation how many patients with 'normal' manometry can be shown to have a functional abnormality. In a recent series ${ }^{1+}$ radionuclide transport was used to study oesophageal function in a group of patients with dysphagia but with 'normal' manometry. Transit disorders were seen in more than half of this group. In another more recent series ${ }^{13}$ a lower incidence was noted, and this might further support the hypothesis that measurements of absolute values of peristaltic amplitude, 
velocity and duration do not reliably predict the functional status of the oesophagus.

Our present study does show a small intraindividual variation with time when the site of measurement within the oesophagus remains constant. This means that oesophageal manometry can be used to assess the effect of drugs or other pharmacologic agents on oesophageal peristalsis. This is only valid where each subject is used as his or her own control and only measurements of amplitude, velocity and duration at the same level before and after therapeutic manipulation are compared. Subjects (such as subject 4 in our study) with a large inherent variation may not be suitable for inclusion in such studies. If absolute values of peristalsis have no predictive value regarding function then altering these values by pharmacologic manipulation should have no effect on function. Humphries and Castell" showed that oral bethanecol produced a significant increase in amplitude and a decrease in velocity values $(p<0 \cdot 05)$. They surmised that oral bethanecol might therefore produce 'a stronger but slower, perhaps more efficient, progression of the peristaltic wave'. Phaosawasdi et al" in a similar study confirmed these findings in amplitude and velocity after bethanecol. They also studied the effect of bethanecol on radionuclide transport and the results showed that bethanecol had no significant effect on transit - that is, did not enhance it, even in patients with abnormal transit before therapeutic manipulation. This is further evidence that absolute values of peristaltic parameters do not necessarily predict function.

What investigative method does predict function? There is no doubt that the oesophageal peristaltic wave is a major component of deglutition. Manometry with a continuous perfusion side hole system, as was used in the studies quoted above, relies on occlusion of the side hole by the mucosa during peristalsis to produce a pressure rise within the system. Assuming total occlusion and noncompliance the pressure within the system will rise till it equals the pressure of the oesophageal contraction. This occlusive force is a circumferential 'squeeze'. The physical forces necessary to drive a bolus down the oesophagus need to be considered. The 'resultant' force vector must of necessity work in an aboral direction. This 'resultant' or aboral force can be and has been measured in the past. ${ }^{15}{ }^{16}$ Both these studies used a linear strain gauge which could measure the distracting force on a detachable sphere. One study ${ }^{15}$ showed that the relationship between pressure and force, although positive, was weak and the other ${ }^{16}$ stated that peristaltic amplitude 'was a good index of peristaltic force'. In the latter study, ${ }^{16}$ however, they concluded 'The determination of intraluminal oesophageal pressure did not accurately reflect the differences in force at each oesophageal level and with different bolus sizes'. This observation suggests that force may be determined by factors that are not wholly reflected in the pressure (squeeze) measurements. It remains to be seen whether oesophageal force measurements are a better measure of function. We are currently investigating this possibility.

Is there a clinical message from this statistical exercise? We feel there is and suggest the following principles: (a) manometric measurements are of diagnostic value where peristalsis is absent or infrequent; (b) when peristalsis is present one should be guarded about predicting abnormality from absolute values; (c) if clinicians insist on the importance of absolute values, then the control range must be drawn from their own normal population studied by their own equipment. The range would more appropriately be mean $\pm 3 \mathrm{SD}$; (d) studies on the effects of drugs and hormones on manometry are valid provided; (e) each subject is used as his own control; (f) our studies indicate values obtained from the mid oesophagus will be most reliable. Information from other sites may be difficult to interpret; (g) subjects with a wide intra-individual variation in values - for example, subject 4 , should be excluded.

\section{References}

1 Pope C. Effect of infusion on force of closure measurements in the human esophagus. Gastroenterology 1970; 58: 616-24.

2 Arndorfer R, Stef J, Didds W, et al. Improved infusion system for intraluminal esophageal manometry. Gastroenterology 1977; 73: 23-7.

3 Dent J. A new technique for continuous sphincter pressure measurement. Gastroenterology 1976; 71: 263-7.

4 Dent J, Culross $\mathbf{J}$, Morris $\mathbf{J}$. A pneumatically driven pump for constant perfusion manometry. Aust J Exp Biol Med Sci 1977; 55: 293-8.

5 Evanden A, Dowlatshahi K. Distal esophageal and lower esophageal sphincternometry in healthy volunteers. Surg Gastroenterol 1983; 2: 253-9.

6 Ask P, Tibbling L. Effect of time intervals between swallows on esophageal peristalsis. Am J Physiol 1980; 238: $485-90$

7 Hollis J, Castell D. Effect of dry swallows and wet swallows of different volumes on esophageal peristalsis. J Appl Physiol 1975; 38: 1161-4.

8 Benjamin S, Gerhardt D, Castell D. High amplitude peristaltic esophageal contractions associated with chest pain and/or dysphagia. Gastroenterology 1979; 77: 478-83.

9 Dodds W, Dent J, Hogan W, et al. Effect of atrophine on esophageal motor function in humans. Am J Physiol 1981 240: 290-6.

10 Humphries T, Castell D. Effect of oral bethanecol on 
parameters of esophageal peristalsis. Dig Dis Sci 1981; 26: $129-32$.

11 Phaosawasdi K, Malmud L, Tolin R, et al. Cholinergic effects on esophageal transit and clearance. Gastroenterology 1981; 81: 915-20.

12 Benjamin S, O’Donnel J, Hancock J, et al. Prolonged radionuclide transit in 'nutcracker oesophagus'. Dig Dis Sci 1983; 28: 775-9.

13 De Caestecker J, Blackwell J, Adam R, et al. Clinical value of radionuclide oesophageal transit measurement.
Gut 1986; 27: 659-66.

14 Russell C, Hill L, Holmes E, et al. Radionuclide transit: a sensitive screening test for esophageal dysfunction. Gastroenterology 1981; 80: 887-92.

15 Pope $C$, Horton $P$. Intraluminal force transducer measurements of human oesophageal peristalsis. Gut 1982; 13: 464-70.

16 Schoen H, Morris D, Cohen S. Esophageal peristaltic force in man. Response to mechanical and pharmacological alterations. Dig Dis Sci 1977; 22: 589-97. 